Bull. Korean Math. Soc. 49 (2012), No. 5, pp. 961-987

http://dx.doi.org/10.4134/BKMS.2012.49.5.961

\title{
VALVELESS PUMPING IN OPEN TANK SYSTEM USING ENERGY CONSERVING COMPARTMENT MODEL
}

\author{
EUNOK JUng AND Do WAN Kim
}

\begin{abstract}
A compartment model of the flow driven by pumping without valves (valveless pumping) in an open tank system is proposed. By the open tank system, we mean that two rigid cylindrical tanks are connected with an elastic tube. An incompressible fluid fills this system up to a certain level in tanks under the gravity. The compartment model for analyzing such open system is derived from the energy principle which will be called the energy conserving compartment model or shortly the ECCM. Based on this ECCM of valveless pumping, we explore the occurrence of directional net flow or directional net power by a specific excitation at an asymmetric part of the elastic tube. The interaction between deformable elastic tube and the fluid inside is considered in the ECCM. The reliability of the ECCM is investigated through some physical examples. The ECCM shows the existence of directional net power of the valveless pump system with open tanks and confirms that the direction and magnitude of the net power depend on the pumping frequency as well. Furthermore, the phase synchronization in time between the fluid pressure difference and the external pinching force over the pumping region is highly related to the direction of energy storing or net power.
\end{abstract}

\section{Introduction}

The valveless pumping, the Liebau effect, and the impedance pumping describe the common phenomenon that a net power can be made by simple periodic excitation on an asymmetric part of an elastic tube filled with an incompressible fluid. Recently, there have been trials to apply this phenomenon to micro pumping devices $[5,11,25]$ or to understand the valveless pumping mechanism itself [2]. In case of a closed loop system of valveless pumping, this net power causes a net flow in a preferential direction, while it can store the potential energy in an open tank system under gravity. In this paper, we consider an open valveless pump system that consists of two open rigid tanks

Received May 12, 2011; Revised August 24, 2011.

2010 Mathematics Subject Classification. 97M10, 81T80, 82C21.

Key words and phrases. valveless pumping, compartment model, energy principle, energy storing, net power, phase synchronization.

The first author was supported by Konkuk University Research Grant in 2010.

The second author was supported by Inha University Research Grant in 2010. 
with a connecting elastic tube and develop the energy conserving compartment model (ECCM) of the system using the energy principle.

To understand the mechanism of valveless pumping phenomena discussed by Liebau [14], many attempts have been made for recent decades in theoretical [1, 17, 21], experimental [4, 7, 8, 20, 22], and numerical [3, 6, 9, 12, 13] point of view. Kenner [10] points out that the energy-driven source of fluid movement, asymmetry of conduits, and an asymmetry of the course of movement are conditions for valveless pumping. In closed loop systems, Bringley et al. [4] consider a closed loop model composed of an elastic tube and a rigid tube to investigate the Liebau phenomenon under the low frequency of external pumping. Similarly, in a closed loop system of a soft and a rigid tube, Manopoulos [17] has derived a nonlinear hyperbolic partial differential equation to consider the flow separation hydraulic losses at the divergent part of the stenosis at the excitation region. Using the fluid-structure interaction formulation, Loumes et al. [16] has calculated a multi-layer impedance pumping system based on the resonance excitation. Olsson [19] has computed a lumped mass model for valveless diffuser pump. Hikerson [8] has demonstrated that the net flow rate is highly sensitive to pumping frequency, which has been commonly observed by other researchers.

In case of an open valveless pumping system which consists of fluid tanks and the connecting elastic tubes or rigid pipes, there are marked results by Takagi, Propst, and Borzi. Takagi [23] reports that valveless pumping can take place in real experiment and numerical simulations by proposing a model of rigid pipes. Recently, Propst [21] proposed the necessary and sufficient conditions for pumping on the nonlinear models of rigid pipe and fluid tanks under gravity. Borzi [3] and Propst [21] have performed the comparison study among models of open tank systems (an elastic tube and a rigid pipe) by means of numerical simulations. They pay attention to the conditions under which valveless pumping takes place.

The modeling and numerical computations become inevitable parts as tools to analyze the mechanism of valveless pumping. There seems to be three important ways to do that: first, using a specific averaging technique and modeling the viscous effect, three-dimensional incompressible Navier-Stokes equations are reduced to a one-dimensional hyperbolic type of partial differential equation under certain assumptions. Second, using the immersed boundary method or numerical packages, the interaction problem between the incompressible Navier-Stokes flow and the elastic tube is directly solved. Third, regarding the entire fluid as a combination of lumps of fluid, a system of first order ordinary differential equations for lumps of fluid is considered. Despite the fact that much effort successfully has revealed the mechanism to explain the occurrence of a directional net flow (or power) in valveless pumping, we still need to seek the causes of the pumping direction change according to the external pumping frequency. 
Based on the energy principle, a reliable compartment model of the open valveless pump system under gravity is developed. The equations derived from the energy principle on each compartment of the system thus satisfy the energy conserving property in the entire system, so that it is called the energy conserving compartment model or briefly the ECCM. It is motivated by Jung's lumped model [9] which is a lumped model for a closed loop system of valveless pumping. Based on the Ohm's law and the definition of compliance, Jung's model is designed for the flow system in a closed loop with soft and stiff parts on an elastic tube. However, the ECCM employs only the energy principle together with the constitutive law between the radial strain and its corresponding pressure of the elastic tube under some feasible assumptions. Although Ottesen [20, 24] and Manopoulos [18] have used a constitutive law similar to the ECCM, taking care of the time-dependent wall thickness of the elastic tube is markedly different from them. Since the wall thickness plays a role in strengthening Young's modulus, time dependent thickness of the tube wall should be considered in our model.

In the most recent modeling of the open tank system by Timmermann and Ottesen [24], using the non-stationary Bernoulli principle along a streamline in a tank, boundary conditions for the dimensionless first order hyperbolic system are introduced. To validate their model, they compare the gravitationally driven oscillating flow with experiment data. They added to a marked explanation for the mechanism for valveless flow in an open tank system by introducing the horizontal slope frequencies. In the same spirit, we first show the reliability of the ECCM. Physical phenomena including the hydrostatic pressure are revived using the ECCM. Additionally, it is proved that the entire energy over the system is conserved by introducing the elastic energy of the tube, the energy caused by the external pumping, and the energy decaying by the friction force on the tube wall. It is shown numerically that the magnitude of a stored energy including its direction is proportional to the difference of fluid levels in both tanks. Particularly, of interest is to find out that the direction of energy storing is closely related to the phase synchronization between the pressure difference and the external pinching force over the pumping region.

Main topics are addressed in the following order: Section 2 describes the derivation of the ECCM of valveless pumping in the open tank system under gravity. The reliability and energy conserving property of our ECCM is investigated and validated in Section 3. Section 4 is written for the discussion on numerical results of valveless pumping effect. Conclusions are written in Section 5 .

\section{Energy conserving compartment model of valveless pumping in the open tank system}

The schematic diagram of the open valveless pump system is illustrated in Fig. 1. There are two cylindrical tanks, $C_{L}$ and $C_{R}$, with one end open and the 
corresponding cross sectional areas, $A_{L}$ and $A_{R}$, respectively. Both tanks have rigid walls and fluid levels, $H_{L}(t)$ and $H_{R}(t)$ at time $t$. The fluid inside tanks is assumed to be affected downward by gravity $g$. Two rigid open tanks are connected with a cylindrical elastic tube which has a finite wall thickness and preserves its volume during deformation. An incompressible fluid fills the entire system and the fluid motions are generated by a periodic pumping applied on an asymmetric location of the elastic tube.

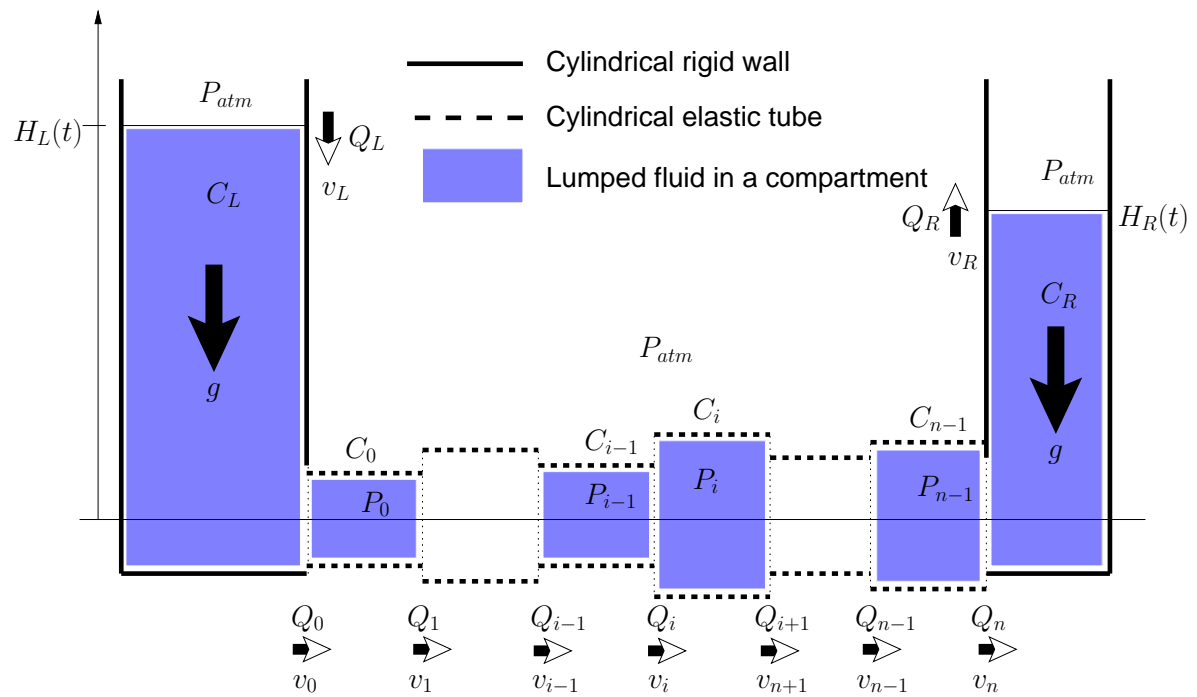

FiguRE 1. Schematic diagram of the proposed compartment model with two open tanks under gravity: $C_{i}$ denotes $i$-th compartment of elastic tube, $P_{i}$ is the pressure in the lumped fluid in the corresponding compartment $C_{i}, Q_{i-1}$ and $Q_{i}$ represent flux respectively at the left and right inlets of $C_{i}$, and $v_{i}$ is the representative velocity corresponding to $Q_{i}$. Particularly, the subscripts, $L$ and $R$ in the form of $\bullet_{L}$ and $\bullet_{R}$ means the correspondence to the left and right tank. For example, $v_{L}$ and $v_{R}$ are the representative velocity of fluids at the left and right tanks, respectively.

Let $n$ denote the number of compartments of the elastic tube, $C_{i}$ for $i=$ $0,1, \ldots, n-1$ be $i$-th compartment, $P_{i}$ for $i=0,1, \ldots, n-1$ the representative pressure of fluid in the compartment $C_{i}$, and $Q_{i}$ for $i=1, \ldots, n-1$ the flux between $C_{i-1}$ and $C_{i}$ as illustrated in Fig. 1. Particularly, $Q_{0}$ and $Q_{n}$ are flux between $C_{L}$ and $C_{0}$ and between $C_{n-1}$ and $C_{R}$, respectively.

To develop the compartment model of valveless pumping with two open tanks under gravity, it is important that the physically meaningful flux conditions have to be derived at two junctions between the elastic tube and two 
tanks. For the purpose of deriving such conditions, the principle of energy transfer will be employed, i.e., the time rate of change in energy (or work) of the lumped fluid inside a compartment is equivalent to the power done by it.

We assume that the flow direction moving from the left to the right tank is defined as positive. In line with this assumption, it should be emphasized that the force acting on a lump of fluid in the compartment model is assumed only one dimensional. This means that the elastic force acting on the lumped fluid has to be transferred to the fluid pressure via the volume change of the incompressible fluid. This feature is revealed well in the middle of verifying the energy conserving property in Section 3.

With the energy principle, the flux between adjacent elastic compartments will be modeled first in sub-Section 2.1 and then secondly the motion of fluid between the tank and its connected elastic compartment will be modeled in sub-Section 2.2. In sub-Section 2.3, using the elastic force generated by the radial displacement of a cylindrical tube with finite length, we also derive the relationship between the pressure of fluid and the radius of the cylindrical tube in the same elastic compartment under the condition that the mass of tube is negligible.

\subsection{Flux model between adjacent compartments in the elastic tube}

We consider the fluid flow $Q_{i}(t)$ between two consecutive compartments in the elastic tube, $C_{i-1}$ and $C_{i}$ for $i=1, \ldots, n-1$. The length of each compartment $C_{i}$ is denoted by $l_{i}$. Assume that $v_{i}$ represents the velocity corresponding to $Q_{i}$ and $A_{i}$ designates the cross sectional area of the lumped fluid in $C_{i}$.

Let us consider the control compartment $\bar{C}_{i}=C_{i-1}^{+} \cup C_{i}^{-}$where $C_{i}^{-}$and $C_{i}^{+}$denote the left and right halves of $i$-th compartment $C_{i}$, respectively. The conceptual diagram for $\bar{C}_{i}$ is illustrated in Fig. 2. We define a fluid volume $\bar{V}_{i}$ in $\bar{C}_{i}$ as

$$
\bar{V}_{i} \equiv \frac{1}{2} l_{i-1} A_{i-1}+\frac{1}{2} l_{i} A_{i}=\frac{1}{2}\left(V_{i-1}+V_{i}\right)
$$

which is regarded as an artificial volume of fluid associated with the flux $Q_{i}$. Then, the representative velocity of the fluid in $\bar{V}_{i}$ can be defined as

$$
v_{i} \equiv \frac{Q_{i}}{\bar{A}_{i}},
$$

where the representative area $\bar{A}_{i}$ for $\bar{V}_{i}$ has to be defined as follows

$$
\bar{A}_{i} \equiv \frac{l_{i-1} A_{i-1}+l_{i} A_{i}}{l_{i-1}+l_{i}}
$$

since the volume of $\bar{V}_{i}$ has to equal that of the fluid in $\bar{C}_{i}$. From the definitions, (1) and (3), we can obtain the following identities

$$
\bar{V}_{i}=\bar{A}_{i} \bar{l}_{i}, \quad \bar{l}_{i} \equiv \frac{1}{2}\left(l_{i-1}+l_{i}\right)
$$


On the other hand, since the fluid is assumed to be incompressible, the volume conservation of fluid can be written in the following form

$$
\dot{V}_{i}=Q_{i}-Q_{i+1}, \quad i=0,1, \ldots, n-1,
$$

provided $V_{i} \equiv A_{i} l_{i}$, the volume of fluid in the compartment $C_{i}$.
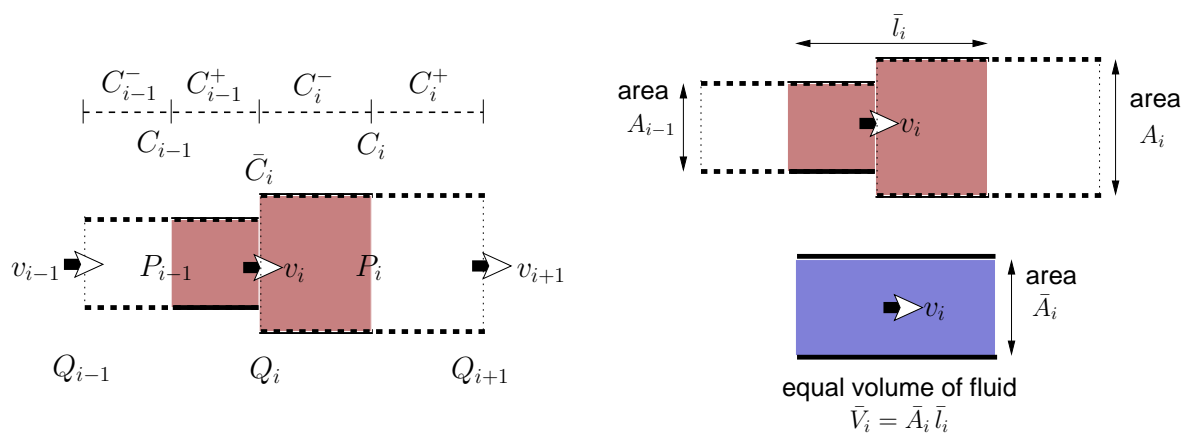

Figure 2. Compounded (or control) compartment $\bar{C}_{i}$ between $C_{i-1}$ and $C_{i}$ : $\bar{V}_{i}, \bar{A}_{i}$, and $\bar{l}_{i}$ are the equal volume to $\bar{C}_{i}$, the cross sectional area of the equal volume, and the length of $\bar{C}_{i}$, respectively.

If $e_{i}$ stands for the kinetic energy of the fluid in $\bar{C}_{i}$, then the volume and velocity of fluid in $\bar{C}_{i}$, defined respectively in (1) and (2), leads to the following representation for $e_{i}$

$$
e_{i}=\frac{1}{2} \rho \bar{V}_{i} v_{i}^{2}=\frac{1}{2} \rho Q_{i}^{2} \frac{\bar{l}_{i}^{2}}{\bar{V}_{i}}
$$

Considering the viscosity effect, we introduce the friction force $\mathcal{F}_{i}$ exerted on the wall of the cylindrical tube by the fluid with volume $\bar{V}_{i}$ and it is transformed to an equivalent pressure difference on $\bar{A}_{i}$ approximated by Hagen-Poiseuille laminar flow, i.e.,

$$
\mathcal{F}_{i}=8 \pi \mu \bar{l}_{i} v_{i}
$$

where $\mu$ is the dynamic viscosity of fluid.

We need here to remark something about the compartment model. The kinetic energy $e_{i}$ is apparently affected by the elastic force from the tube which is applied perpendicularly to the inside fluid in the compounded compartment $\bar{C}_{i}$. Since the fluid in the compartment model has only one-dimensional forces, the elastic force has to be embedded into the fluid pressure in a reasonable way. Under some assumptions, the relationship between the fluid pressure and the elastic pressure induced by the tension of the elastic tube can be modeled. Details are addressed in sub-Section 2.3. It should be pointed out that the fluid pressure $P_{i}$ is the uni-directional pressure along the compartment. 
From the relationship between the energy and power of the fluid in $\bar{C}_{i}$, we have

$$
\begin{aligned}
\dot{e}_{i} & =-\left(P_{i} \bar{A}_{i}\right) v_{i}+\left(P_{i-1} \bar{A}_{i}\right) v_{i}-\mathcal{F}_{i} v_{i} \\
& =\left(P_{i-1}-P_{i}\right) \bar{A}_{i} v_{i}-8 \pi \mu \bar{l}_{i} v_{i}^{2} \\
& =\left(P_{i-1}-P_{i}\right) Q_{i}-8 \pi \mu Q_{i}^{2} \frac{\bar{l}_{i}}{\bar{A}_{i}^{2}} .
\end{aligned}
$$

Due to the incompressibility of the fluid in (5) and the definition (1) of the fluid volume inside $\bar{C}_{i}$, the time rate of change for $e_{i}$ in (6) becomes

$$
\begin{aligned}
\dot{e}_{i} & =\rho Q_{i} \dot{Q}_{i} \bar{\Delta}_{i}+\frac{1}{2} \rho Q_{i}^{2} \bar{l}_{i}^{2}\left(-\frac{\dot{\bar{V}}_{i}}{\bar{V}_{i}^{2}}\right) \\
& =\rho Q_{i} \dot{Q}_{i} \bar{\Delta}_{i}-\frac{1}{4} \rho Q_{i}^{2}\left(Q_{i-1}-Q_{i+1}\right) \frac{\bar{\Delta}_{i}}{\bar{V}_{i}},
\end{aligned}
$$

provided we define

$$
\bar{\Delta}_{i} \equiv \frac{\bar{V}_{i}}{\bar{A}_{i}^{2}}
$$

As a consequence of (8) and (9), the flux $Q_{i}$ satisfies

$$
\dot{Q}_{i}=\frac{1}{\rho \bar{\Delta}_{i}}\left(P_{i-1}-P_{i}\right)+\frac{1}{4 \bar{V}_{i}} Q_{i}\left(Q_{i-1}-Q_{i+1}\right)-\frac{8 \pi \mu}{\rho \bar{A}_{i}} Q_{i}
$$

for $i=1,2, \ldots, n-1$.

\subsection{Flux model between the tank and elastic tube}

Two rigid tanks are attached to the marginal compartments, $C_{0}$ and $C_{n-1}$, located at both ends of the elastic tube. The levels of fluid in $C_{L}$ and $C_{R}$ are denoted by $H_{L}(t)$ and $H_{R}(t)$, respectively. We shall derive the conditions for the flux, $Q_{0}(t)$ and $Q_{n}(t)$, at the bottom outlets of tanks to the tube. In tanks, assume that the inertia force of the fluid is dominant enough to ignore the friction force due to viscosity. In addition, the fluid in a tank is assumed to move up and down with the same velocity as if it is a single body, i.e., the velocities of the lump of fluid in tanks, $v_{L}(t)$ and $v_{R}(t)$, are functions of time $t$ only for the incompressibility of the fluid and the rigidity of cylindrical tanks.

In the similar way as in Section 2.1, we define compounded compartments, $\bar{C}_{L} \equiv C_{L} \cup C_{0}^{-}$and $\bar{C}_{R} \equiv C_{n-1}^{+} \cup C_{R}$. Then, the total energy $e_{L}$ of the fluid in $\bar{C}_{L}$ becomes

$$
e_{L}=\int_{0}^{H_{L}(t)} \frac{1}{2} \rho v_{L}^{2}(t) A_{L} d y+\int_{0}^{H_{L}(t)} \rho g y A_{L} d y+\frac{1}{4} \rho V_{0} v_{0}^{2},
$$

where $g$ designates the gravitational constant. Actually, the terms on the right hand side represent the kinetic energy of fluid in $C_{L}$, the potential energy of 
fluid in $C_{L}$, and the kinetic energy of fluid in $C_{0}^{-}$in order. Similarly, for the fluid in $\bar{C}_{R}$, we have the energy $e_{R}$

$$
e_{R}=\int_{0}^{H_{R}(t)} \frac{1}{2} \rho v_{R}^{2}(t) A_{R} d y+\int_{0}^{H_{R}(t)} \rho g y A_{R} d y+\frac{1}{4} \rho V_{n-1} v_{n}^{2} .
$$

Using the relationship that

$$
Q_{0}(t)=A_{0} v_{0}=A_{L} v_{L}(t)
$$

we can calculate the terms on the right hand side in (12) to be

$$
\begin{aligned}
e_{L} & =\frac{1}{2} \rho A_{L} H_{L}(t) v_{L}^{2}(t)+\frac{1}{2} \rho g A_{L} H_{L}^{2}(t)+\frac{1}{4} \rho Q_{0}^{2}(t) \frac{l_{0}}{A_{0}(t)} \\
& =\frac{1}{2} \rho Q_{0}^{2}(t)\left[\frac{H_{L}(t)}{A_{L}}+\frac{l_{0}}{2 A_{0}(t)}\right]+\frac{1}{2} \rho g A_{L} H_{L}^{2}(t) .
\end{aligned}
$$

Similarly, the energy $e_{R}$ in (13) can be rewritten as

$$
\begin{aligned}
e_{R} & =\frac{1}{2} \rho A_{R} H_{R}(t) v_{R}^{2}(t)+\frac{1}{2} \rho g A_{R} H_{R}^{2}(t)+\frac{1}{4} \rho Q_{n}^{2}(t) \frac{l_{n-1}}{A_{n-1}(t)} \\
& =\frac{1}{2} \rho Q_{n}^{2}(t)\left[\frac{H_{R}(t)}{A_{R}}+\frac{l_{n-1}}{2 A_{n-1}(t)}\right]+\frac{1}{2} \rho g A_{R} H_{R}^{2}(t)
\end{aligned}
$$

using the analogous formula to (14),

$$
Q_{n}(t)=A_{n-1}(t) v_{n}(t)=A_{R} v_{R}(t) .
$$

Furthermore, the representative fluid velocities in two tanks must satisfy

$$
v_{L}(t)=-\dot{H}_{L} \text { and } v_{R}(t)=\dot{H}_{R},
$$

so that the incompressibility in (5) leads to the time rate of changes for $e_{L}$ and $e_{R}$,

$$
\begin{aligned}
\dot{e}_{L} & =\rho Q_{0} \dot{Q}_{0} \bar{\Delta}_{L}+\frac{1}{2} \rho Q_{0}^{2}\left[\frac{\dot{H}_{L}}{A_{L}}-\frac{\dot{V}_{0}}{2 A_{0}^{2}}\right]+\rho g A_{L} H_{L} \dot{H}_{L} \\
& =\rho Q_{0} \dot{Q}_{0} \bar{\Delta}_{L}+\frac{1}{2} \rho Q_{0}^{2}\left[-\frac{v_{L}}{A_{L}}-\frac{\dot{V}_{0}}{2 A_{0}^{2}}\right]-\rho g H_{L} Q_{0} \\
& =\rho Q_{0} \dot{Q}_{0} \bar{\Delta}_{L}-\frac{1}{2} \rho Q_{0}^{2}\left[\frac{Q_{0}}{A_{L}^{2}}+\frac{Q_{0}-Q_{1}}{2 A_{0}^{2}}\right]-\rho g H_{L} Q_{0}
\end{aligned}
$$

where

$$
\bar{\Delta}_{L}(t)=\frac{V_{L}(t)}{A_{L}^{2}}+\frac{V_{0}}{2 A_{0}^{2}(t)}
$$

and

$$
\dot{e}_{R}=\rho Q_{n} \dot{Q}_{n} \bar{\Delta}_{R}+\frac{1}{2} \rho Q_{n}^{2}\left[\frac{\dot{H}_{R}}{A_{R}}-\frac{\dot{V}_{n-1}}{2 A_{n-1}^{2}}\right]+\rho g A_{R} H_{R} \dot{H}_{R}
$$




$$
\begin{aligned}
& =\rho Q_{n} \dot{Q}_{n} \bar{\Delta}_{R}+\frac{1}{2} \rho Q_{n}^{2}\left[\frac{v_{R}}{A_{R}}-\frac{\dot{V}_{n-1}}{2 A_{n-1}^{2}}\right]+\rho g H_{R} Q_{n} \\
& =\rho Q_{n} \dot{Q}_{n} \bar{\Delta}_{R}-\frac{1}{2} \rho Q_{n}^{2}\left[-\frac{Q_{n}}{A_{R}^{2}}+\frac{Q_{n-1}-Q_{n}}{2 A_{n-1}^{2}}\right]+\rho g H_{R} Q_{n},
\end{aligned}
$$

where

$$
\bar{\Delta}_{R}(t)=\frac{V_{R}(t)}{A_{R}^{2}}+\frac{V_{n-1}}{2 A_{n-1}^{2}(t)} .
$$

It is of worth to note that sign changes take place at two terms between (19) and (21) due to the definition of positive direction of flows in our setting which is shown in (18).

The time rate of change for the energy $e_{L}$ equals the work per unit time done by the surrounding air pressure $P^{a t m}$ on the top surface of the tank and the fluid pressure $P_{0}$ at $C_{0}^{-}$as well as the friction force on the tube wall in $C_{0}^{-}$ as in (8), i.e., on the compounded compartment $\bar{C}_{L}$,

$$
\begin{aligned}
\dot{e}_{L} & =-\left(P_{0} A_{0}\right) v_{0}+\left(P^{a t m} A_{L}\right) v_{L}-8 \pi \mu Q_{0}^{2} \frac{l_{0}}{2 A_{0}^{2}} \\
& =\left(-P_{0}+P^{a t m}\right) Q_{0}-8 \pi \mu Q_{0}^{2} \frac{l_{0}}{2 A_{0}^{2}} .
\end{aligned}
$$

Similarly, on the compounded compartment $\bar{C}_{R}$ related to the right tank, we have

$$
\begin{aligned}
\dot{e}_{R} & =\left(P_{n-1} A_{n-1}\right) v_{n-1}-\left(P^{a t m} A_{R}\right) v_{R}-8 \pi \mu Q_{n}^{2} \frac{l_{n-1}}{2 A_{n-1}^{2}} \\
& =\left(P_{n-1}-P^{a t m}\right) Q_{n}-8 \pi \mu Q_{n}^{2} \frac{l_{n-1}}{2 A_{n-1}^{2}} .
\end{aligned}
$$

On the flux $Q_{0}(t)$, comparing (19) and (23), we conclude

$$
\begin{aligned}
\dot{Q}_{0}= & \frac{1}{\rho \bar{\Delta}_{L}}\left[P^{a t m}-P_{0}(t)+\rho g H_{L}(t)\right] \\
& +\frac{1}{4} \frac{Q_{0}}{\bar{\Delta}_{L}}\left[\frac{2 Q_{0}}{A_{L}^{2}}+\frac{Q_{0}-Q_{1}}{A_{0}^{2}}\right]-\frac{4 \pi \mu}{\rho \bar{\Delta}_{L}} Q_{0} \frac{l_{0}}{A_{0}^{2}},
\end{aligned}
$$

while the equations of (21) and (24) yield the following model for $Q_{n}(t)$ :

$$
\begin{aligned}
\dot{Q}_{n}= & \frac{1}{\rho \bar{\Delta}_{R}}\left[-P^{a t m}+P_{n-1}(t)-\rho g H_{R}(t)\right] \\
& +\frac{1}{4} \frac{Q_{n}}{\bar{\Delta}_{R}}\left[-\frac{2 Q_{n}}{A_{R}^{2}}+\frac{Q_{n-1}-Q_{n}}{A_{n-1}^{2}}\right]-\frac{4 \pi \mu}{\rho \bar{\Delta}_{R}} Q_{n} \frac{l_{n-1}}{A_{n-1}^{2}} .
\end{aligned}
$$

Based on the energy principle, thus far, we have derived the equations for flux as in (11), (25), and (26). To complete the modeling of valveless pumping, we have to build up equations for the representative fluid pressure in compartments. 


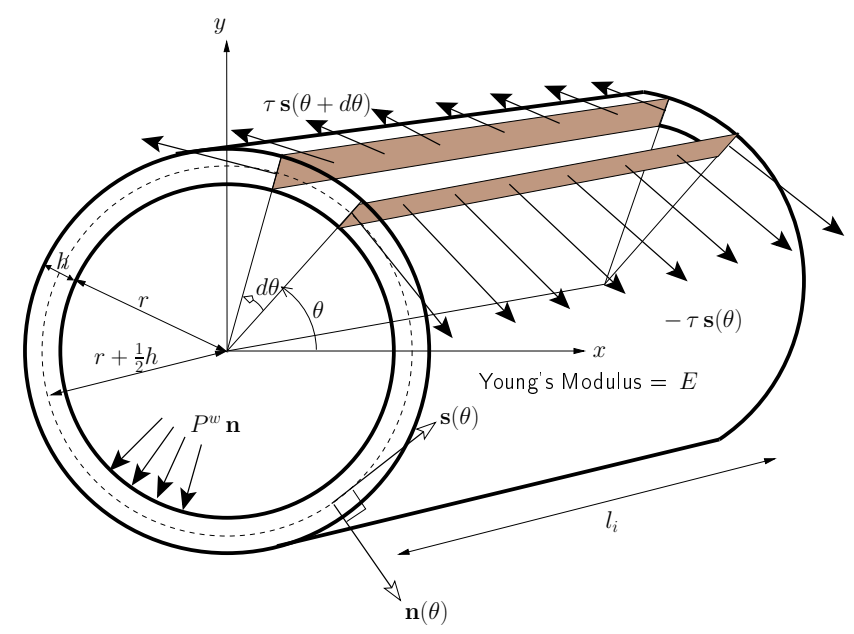

Figure 3. Schematic diagram for the pressure $P^{w}$ induced from the circumferential tension $\tau$ of elastic tube with length $l_{i}$.

\subsection{Pressure model}

The elastic energy ought to apparently contribute somehow to the dynamics of the flow in our model. To understand such a mechanism, we have to reveal how the circumferential tension of the elastic tube is transferred to a part of the fluid pressure inside the elastic tube under some assumptions for the tube as in the reference [15].

Fig. 3 illustrates the structure of the $i$-th compartment $C_{i}$, which consists of a cylindrical elastic tube with thickness $h_{i}(t)$ and Young's modulus $E$ and its cylindrical room with radius $r_{i}(t)$. Let $P^{w}(t)$ be the equilibrium pressure added to the inside wall of the tube induced from the tension $\tau$ of the elastic tube in the compartment $C_{i}$. Along the center circle of the tube cross section, we define the curvilinear coordinate system $\{\mathbf{n}, \mathbf{s}\}$ where $\mathbf{n}$ is the outward unit normal vector and $\mathbf{s}$ is made by rotating $\mathbf{n}$ with angle $\pi / 2$.

To derive the connection equation between $P^{w}(t)$ and the fluid pressure $P(t)$ inside tube, it is assumed during the deformation of the elastic tube that

AS1 the elastic tube in $C_{i}$ maintains its cylindrical shape,

AS2 there is no change in the length $l_{i}$ of $C_{i}$,

AS3 the volume of the elastic tube in $C_{i}$ remains constant,

AS4 the mass of elastic tube in $C_{i}$ is negligible.

In general, the tube initial thickness $h_{i}(0)$ is much smaller than the initial radius $r_{i}(0)$ in valveless pumping, so that the above assumptions, AS1, AS2, AS3, and AS4 seems to be valid. 
We use symbols without subscript for brevity, for example, $r(t)$ instead of $r_{i}(t)$. The first three assumptions, AS1, AS2, and AS3 above induce the following identity for any time $t \geq 0$

$$
(r(t)+h(t))^{2}-r(t)^{2}=D_{0}^{2}
$$

for some constant $D_{0}$ which can be given by the initial conditions.

If the deformation of the tube takes place at infinitesimal time $d t$, then the corresponding infinitesimal variations of $P^{w}, r, h$, and $\tau$ are denoted by $d P^{w}$, $d r, d h$, and $d \tau$, respectively. Considering a small piece of the tube ranging from the angle $\theta$ to $\theta+d \theta$ as depicted in Fig. 3, the following force balance holds

(28) $\left(P^{w}+d P^{w}\right)(r+d r) d \theta l_{i} \mathbf{n}(\theta)+(\tau+d \tau)(\mathbf{s}(\theta+d \theta)-\mathbf{s}(\theta))(h+d h) l_{i}=\mathbf{0}$

since the $P_{w}(t)$ is the balancing force against the circumferential elastic tension $\tau$ and, from our assumptions, the infinitesimal angle $d \theta$ is independent of the variation $d t$ during deformation. Since $d \theta$ is infinitesimal, it is also true that

$$
\mathbf{s}(\theta+d \theta)-\mathbf{s}(\theta)=-\mathbf{n} d \theta .
$$

From the infinitesimal calculus and the identity (29), the equation in (28) results in

$$
\left(P^{w} d r+r d P^{w}\right)-(\tau d h+h d \tau)=0
$$

or, equivalently

$$
d\left(P^{w} r\right)=d(\tau h)
$$

From the definition of Young's modulus, we have

$$
E=\frac{d F / A_{F}}{d \Delta / \Delta}
$$

where $d F$ is the variation of total tension force on the section $A_{F}$ (shadow face in Fig. 3) on which the tension $\tau$ acts under the deformation described above, and $\Delta$ is the length of the center circle of tube cross section before deformation occurs and $d \Delta$ means the variation of $\Delta$ after deformation. The relevant quantities in the definition can be written as

$$
\begin{aligned}
& \frac{d F}{A_{F}}=\frac{(\tau+d \tau)(h+d h) l_{i}-\tau h l_{i}}{h l_{i}}=\frac{d(\tau h)}{h}, \\
& \frac{d \Delta}{\Delta}=\frac{\left((r+d r)+\frac{1}{2}(h+d h)\right) d \theta-\left(r+\frac{1}{2} h\right) d \theta}{\left(r+\frac{1}{2} h\right) d \theta}=d \log \left(r+\frac{1}{2} h\right) .
\end{aligned}
$$

Therefore, as a result of (32), (33), and (34), the following equation is derived

$$
d(\tau h)=E h d \log \left(r+\frac{1}{2} h\right) .
$$


On the other hand, the area conserving condition (27) for the tube cross section produces the differential equation

$$
\left(r+\frac{1}{2} h\right) d h+h d\left(r+\frac{1}{2} h\right)=0, \quad \text { or } \quad(r+h) d h+h d r=0 .
$$

Combining these equivalent equations, we have

$$
d \log \left(r+\frac{1}{2} h\right)=-\frac{1}{h} d h=\frac{d r}{r+h} .
$$

As a consequence of (31), (35), and (37), we obtain the desired equation for $P_{w}(t)$ as follows:

$$
\dot{P}^{w}=\left(\frac{E h}{r+h}-P^{w}\right) \frac{\dot{r}}{r}=\frac{1}{2}\left(\frac{E h}{r+h}-P^{w}\right) \frac{\dot{V}}{V}
$$

provided that $V(t)$ is the volume of the room inside $i$-th compartment $C_{i}$.

If we assume that the fluid pressure inside the compartment $C_{i}$ is denoted by $P_{i}(t)$ and both the atmospheric pressure $P^{a t m}$ and the external pumping force $P_{i}^{e x t}(t)$ are applied to the outside wall of the tube in $C_{i}$, then negligible mass assumption AS4 for the elastic tube implies

$$
P_{i}^{w}(t)=P_{i}(t)-P_{i}^{e x t}(t)-P^{a t m} \text { for } i=0,1, \ldots, n-1 .
$$

Therefore, from the equation (38) and incompressibility (5), the differential equation for $P_{w}$ becomes

$$
\dot{P}_{i}^{w}=\frac{1}{2}\left(\frac{E h_{i}}{r_{i}+h_{i}}-P_{i}^{w}\right) \frac{Q_{i}-Q_{i+1}}{V_{i}}
$$

for $i$-th compartment, $i=0,1, \ldots, n-1$. Here, it should be emphasized that the elastic force and the fluid pressure are mutually transferred to each other via the equations, (39) and (40), resulting from our assumptions.

We have thus far derived sets of first order differential equations for $Q_{i}(t)$, $P_{i}(t), H_{L}(t)$, and $H_{R}(t)$. To complete the system, additional first order differential equations for $r_{i}(t)$ 's are needed to calculate areas and volumes of elastic compartments. Fortunately, it has been given already to satisfy the incompressibility of the fluid in (5).

A system of ordinary differential equations in our energy conserving compartment model can be addressed with the solutions $P_{i}^{w}(t), Q_{i}(t), r_{i}(t), H_{L}(t)$, and $H_{R}(t)$ as follows:

- Pressure equations

$$
\dot{P}_{i}^{w}=\frac{1}{2}\left(\frac{E h_{i}}{r_{i}+h_{i}}-P_{i}^{w}\right) \frac{Q_{i}-Q_{i+1}}{V_{i}}, \quad i=0,1, \ldots, n-1,
$$

- Flux equations

$$
\dot{Q}_{0}=\frac{1}{\rho \bar{\Delta}_{L}}\left[-P_{0}^{w}(t)-P_{0}^{e x t}(t)+\rho g H_{L}(t)\right]
$$




$$
\begin{aligned}
& +\frac{1}{2} Q_{0}\left[\frac{Q_{0}}{V_{L}} \frac{\Delta_{L}}{\bar{\Delta}_{L}}+\frac{Q_{0}-Q_{1}}{V_{0}} \frac{\Delta_{0}}{2 \bar{\Delta}_{L}}\right]-\frac{8 \pi \mu}{\rho} \frac{Q_{0}}{A_{0}} \frac{\Delta_{0}}{2 \bar{\Delta}_{L}}, \\
\dot{Q}_{i}= & \frac{1}{\rho \bar{\Delta}_{i}}\left[P_{i-1}^{w}(t)+P_{i-1}^{e x t}(t)-P_{i}^{w}(t)-P_{i}^{e x t}(t)\right] \\
& +\frac{1}{2} Q_{i} \frac{Q_{i-1}-Q_{i+1}}{2 \bar{V}_{i}}-\frac{8 \pi \mu}{\rho} \frac{Q_{i}}{\bar{A}_{i}}, \quad i=1,2, \ldots, n-1, \\
\dot{Q}_{n}= & \frac{1}{\rho \bar{\Delta}_{R}}\left[P_{n-1}^{w}(t)+P_{n-1}^{e x t}(t)-\rho g H_{R}(t)\right] \\
+ & \frac{1}{2} Q_{n}\left[-\frac{Q_{n}}{V_{R}} \frac{\Delta_{R}}{\bar{\Delta}_{R}}+\frac{Q_{n-1}-Q_{n}}{V_{n-1}} \frac{\Delta_{n-1}}{2 \bar{\Delta}_{R}}\right]-\frac{8 \pi \mu}{\rho} \frac{Q_{n}}{A_{n-1}} \frac{\Delta_{n-1}}{2 \bar{\Delta}_{R}},
\end{aligned}
$$

- Incompressibility equations

$$
\begin{aligned}
\dot{H}_{L} & =-\frac{Q_{0}}{A_{L}}, \quad \dot{H}_{R}=\frac{Q_{n}}{A_{R}}, \\
\dot{r}_{i} & =\frac{r_{i}}{2} \frac{Q_{i}-Q_{i+1}}{V_{i}} \text { for } i=0,1, \ldots, n-1,
\end{aligned}
$$

- Massless condition for the elastic tube

$$
P_{i}(t)=P_{i}^{w}(t)+P^{a t m}+P_{i}^{e x t}(t) \text { for } i=0,1, \ldots, n-1,
$$

- State equation for the elastic tube

$$
h_{i}(t)=\sqrt{D_{0}^{2}+r_{i}^{2}(t)}-r_{i}(t) \text { for } i=0,1, \ldots, n-1,
$$

where the symbols used above are listed as follows:

$$
\begin{aligned}
& \Delta_{L}=\frac{V_{L}(t)}{A_{L}^{2}}, \quad \Delta_{0}=\frac{V_{0}}{A_{0}^{2}(t)}, \\
& \bar{\Delta}_{L}=\frac{V_{L}(t)}{A_{L}^{2}}+\frac{V_{0}}{2 A_{0}^{2}(t)}=\Delta_{L}+\frac{1}{2} \Delta_{0}, \\
& \bar{\Delta}_{i}=\frac{\bar{V}_{i}}{\bar{A}_{i}^{2}}, i=1,2, \ldots, n-1, \\
& \Delta_{R}=\frac{V_{R}(t)}{A_{R}^{2}}, \quad \Delta_{n-1}=\frac{V_{n-1}}{A_{n-1}^{2}(t)}, \\
& \bar{\Delta}_{R}=\frac{V_{R}(t)}{A_{R}^{2}}+\frac{V_{n-1}}{2 A_{n-1}^{2}(t)}=\Delta_{R}+\frac{1}{2} \Delta_{n-1} .
\end{aligned}
$$

Here, we note that the atmospheric pressure $P^{a t m}$ plays no role, so that it can be set zero without loss of generality. Important symbols and notations are listed in Table 1 where all dimensions are represented by CGS unit. 
TABLE 1. Symbols and notations: the values inside bracket are fixed in all computations.

\begin{tabular}{c|l|l}
\hline Symbol & Description & CGS unit \\
\hline \hline$C_{L}\left(C_{R}\right)$ & the compartment of the left (right) tank & \\
$H_{L}(t)\left(H_{R}(t)\right)$ & the fluid level in $C_{L}\left(C_{R}\right)$ & $\mathrm{cm}$ \\
$r_{L}\left(r_{R}\right)$ & the radius of the left (right) tank & $\mathrm{cm}$ \\
$d_{L}\left(d_{R}\right)$ & the diameter of the left (right) tank & $\mathrm{cm}$ \\
$A_{L}\left(A_{R}\right)$ & the cross sectional area of the fluid in $C_{L}\left(C_{R}\right)$ & $\mathrm{cm}^{2}$ \\
\hline$n$ & the number of compartments in elastic tube & \\
$C_{i}$ & the $i$-th compartment of elastic tube from the left tank & \\
$l_{i}$ & the length of $C_{i}$ & $\mathrm{~cm}$ \\
$L$ & the total length of elastic tube & $\mathrm{cm}$ \\
$P_{i}(t)$ & the representative pressure of fluid in $C_{i}\left[P_{i}(0)=0\right]$ & $\mathrm{dyne} / \mathrm{cm}^{2}$ \\
$Q_{i}(t)$ & the flux between fluids in $C_{i-1}$ and $C_{i}\left[Q_{i}(0)=0\right]$ & $\mathrm{cm} / \mathrm{s}$ \\
$r_{i}(t)$ & the radius of fluid in $C_{i}\left[r_{i}(0)=0.5\right]$ & $\mathrm{cm}^{3}$ \\
$h_{i}(t)$ & the thickness of elastic tube in $C_{i}$ & $\mathrm{~cm}^{3}$ \\
\hline$\rho$ & the fluid density [= 1.0] & $\mathrm{g} / \mathrm{cm}^{3}$ \\
$\mu$ & the fluid viscosity [=0.01] & $\mathrm{cm} / \mathrm{s}$ \\
$g$ & the gravitational acceleration $[=980]$ & $\mathrm{dyne} / \mathrm{cm}^{2}$ \\
$E$ & the Young's modulus of the elastic tube &
\end{tabular}

\section{Reliability and energy conserving property of the ECCM}

The ECCM in (41)-(48) is derived in the previous section based on the energy principle under feasible assumptions. Assuming there is no external pumping $\left(P^{e x t}(t)=0\right)$, we attest the validity in the following ways:

[Hydrostatic fluid pressure] If we consider the case that the levels of fluid in both tanks are initially equal, i.e., $H_{L}(0)=H_{R}(0)$ at initial state and the radius of the elastic tube is assumed to be initially constant, $r_{i}(0)=r_{0}$ as if there is no fluid inside, i.e., $P_{i}^{w}(0)=0$, then it is expected that the fluid levels in tanks, $H_{L}(t)$ and $H_{R}(t)$, eventually move down a little to a certain level $H_{\infty}<H_{L}(0)=H_{R}(0)$ at the equilibrium state. Due to the total volume conservation modeled in (45) and (46), the tube has to get a little swollen compared to the initial shape to make up for the loss of fluid volume from two tanks, i.e., $r_{i}(t)$ approaches $r_{\infty}>r_{i}(0)$ for each $i$. In this case, the hydrostatic fluid pressure $P_{\infty}$ inside the elastic tube satisfies

$$
P_{\infty}=P_{i}(\infty)=P^{a t m}+\rho g H_{\infty} .
$$

In fact, it can be justified by the ECCM described in (41)-(46). If the steady state exists, then the equations, (45) and (46) leads to the consequence that $Q_{i}(t)$ approaches 0 for all $i=0,1, \ldots, n$ as time goes on. Consequently, $P_{i}^{w}(t)$ 's reach some constant value $P_{\infty}^{w}$ as anticipated from (41). At the same time, the equations, (42), (43), and (44) enable us to predict that $P_{0}^{w}(t)$ and $P_{n-1}^{w}(t)$ approach $\rho g H_{\infty}$. Since there is no external pumping, we have $P_{i}^{w}(t)=$ $P_{i}(t)-P^{a t m}$ so that the hydrostatic pressure identity in (54) holds. 
(5C) Tanks with equal diameter $d=20$

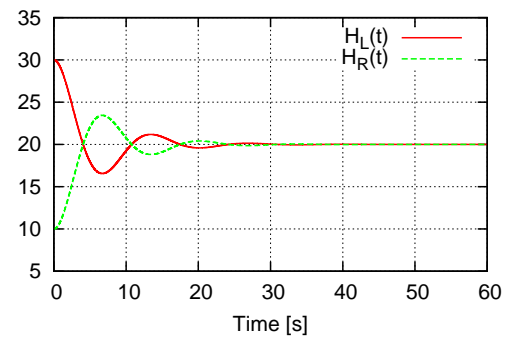

(5C) Tanks with equal diameter $d=40$

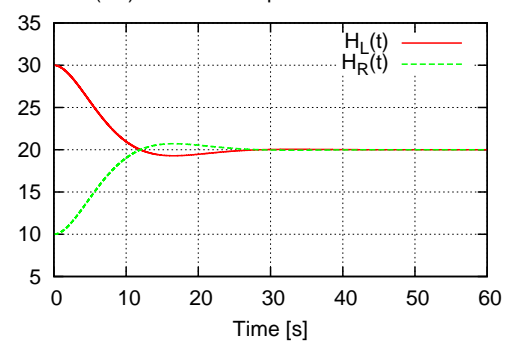

(5C) Tanks with equal diameter $d=60$

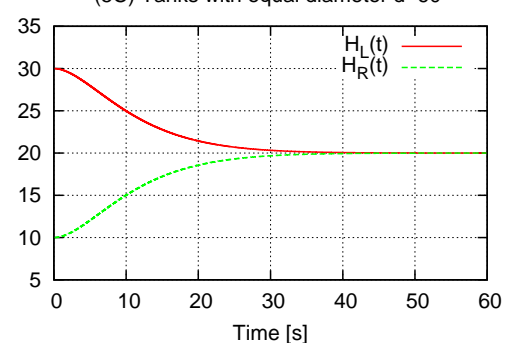

(5C) Tanks with equal diameter $d=80$

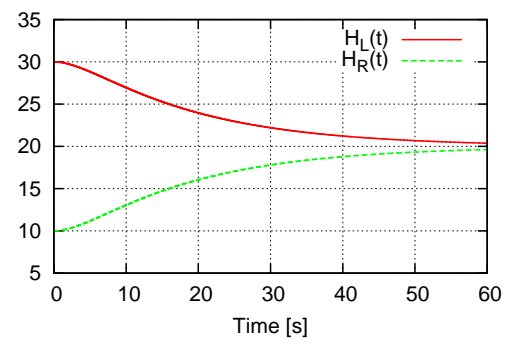

(5C) Flux $Q_{i}$ 's when $d_{L}=d_{R}=20$

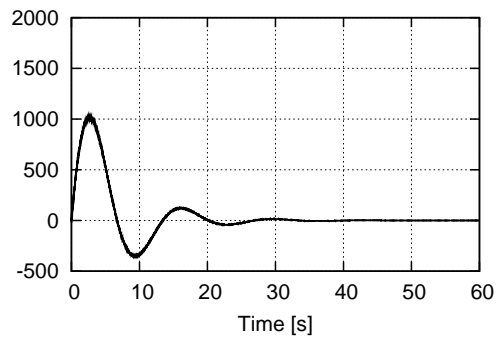

(5C) Flux $Q_{i}^{\prime}$ 's when $d_{L}=d_{R}=40$

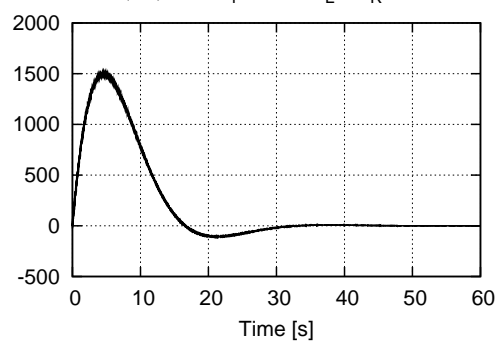

(5C) Flux $Q_{i}$ 's when $d_{L}=d_{R}=60$

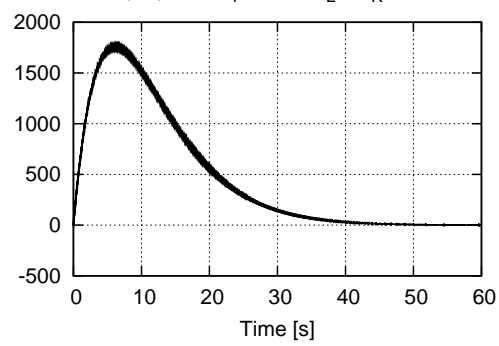

(5C) Flux $Q_{i}$ 's when $d_{L}=d_{R}=80$

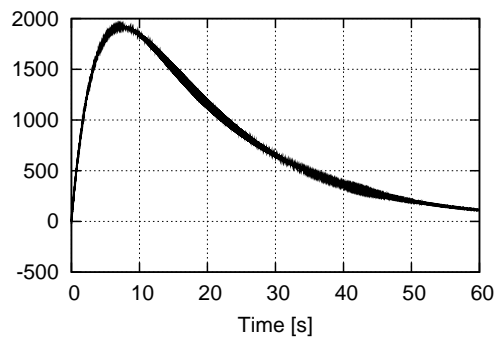

FiguRE 4. Oscillating flows influenced by the tank sizes: tank diameters $d_{L}=d_{R}=20,40,60,80$, the tube length $L=20$ with 5 even compartments $(5 C)$, the Young's modulus $E=1.0 \times 10^{8}$, the initial thickness of tube compartments $h_{i}(0)=0.1$, and the initial heights of fluid in tanks $H_{L}(0)=30, H_{R}(0)=10$. 

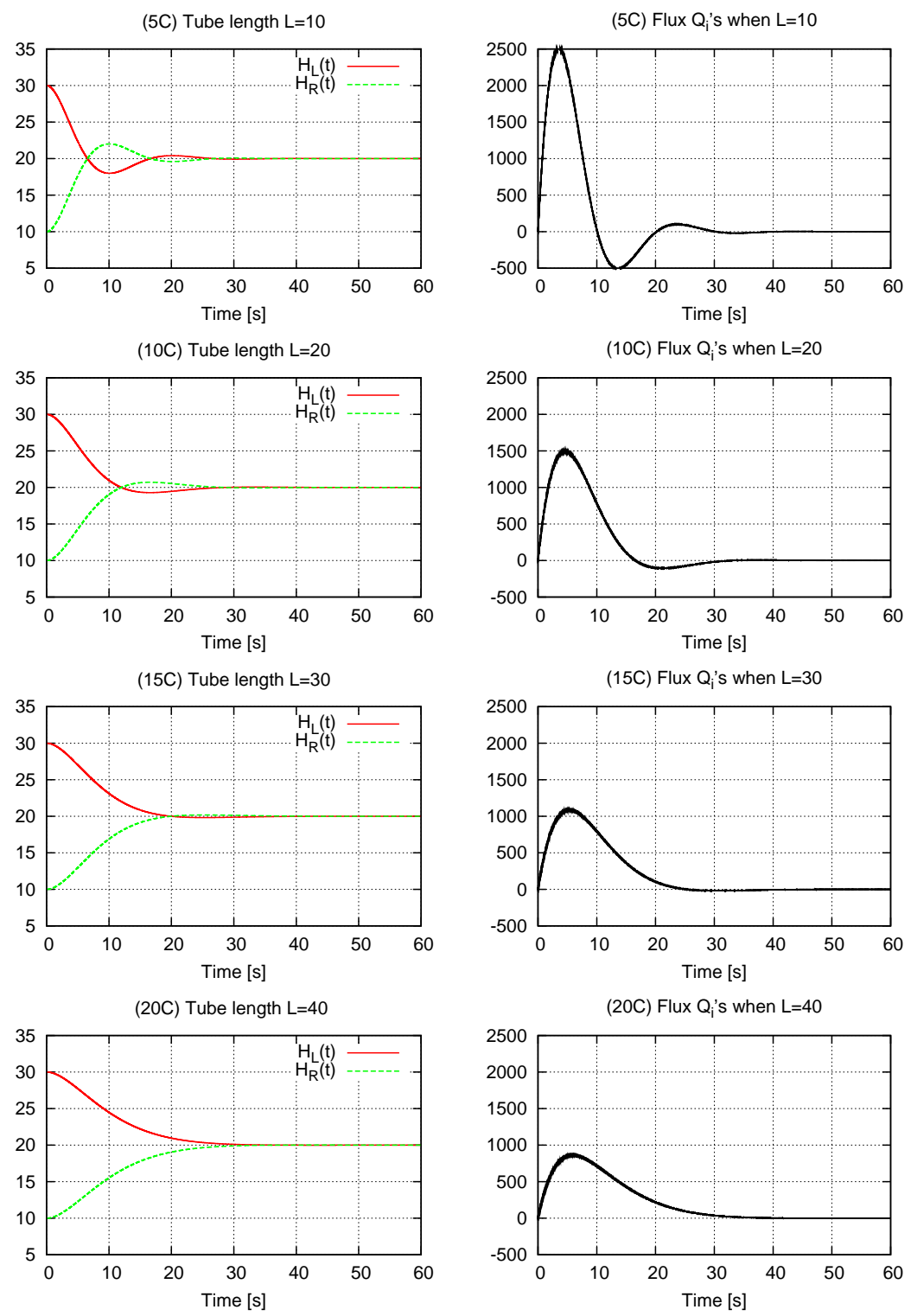

Figure 5. Oscillating flows influenced by the length of elastic tube: the tube length $L=10,20,30,40$ with compartments of length 2 , tank diameters $d_{L}=d_{R}=40$, the Young's modulus $E=1.0 \times 10^{8}$, the initial thickness of tube compartments $h_{i}(0)=0.1$, and the initial heights of fluid in tanks $H_{L}(0)=30, H_{R}(0)=10$. 
[Oscillating flow] Initially different fluid levels in two tanks approaches an equal level at the equilibrium state. If the fluid level in the left tank is initially higher than that in the right tank, then there occur an oscillating flow between two tanks which will stop eventually because of the friction on the tube wall.

We show that the ECCM can realize the oscillating flow between tanks in a variety of situations. The fluid starts moving from the left tank toward the right one at the first instance. The frictional force on the elastic tube wall acts on the moving fluid during oscillations and thus all fluid flux $Q_{i}(t)$ 's decay to zero. In order to show this phenomenon, we first investigate the variation of fluid levels, $H_{L}(t)$ and $H_{R}(t)$, in tanks and its corresponding flux $Q_{i}(t)$ 's as a function of time for various $n$.

If the tank size gets bigger with the same initial tube radius, then the initial frequency of oscillating flow becomes decreasing. In fact, ignoring the friction of the fluid on the wall and assuming the tube is stiff, it can be shown that the frequency of oscillating flow caused by the gravity is approximately proportional to the ratio of radii, $r_{\text {tube }} / r_{\text {tank }}$ as it goes small. This pattern of oscillating flow is shown in Fig. 4. According to the result in Fig. 4, the oscillating frequency decreases when the cross sectional area of tanks is wider but there is almost no oscillating when the equal tank radius is bigger than $30(\mathrm{~cm})$ within 60 seconds. The value of the maximum flux increases as the tank radius gets bigger. On the other hand, we find out in Fig. 5 that regardless of the tube length variation the flow reaches the steady state within 30 seconds in case where $d_{L}=d_{R}=40$. On the contrary to the previous case, the maximum flux decreases as the tube length $L$ increases. This result is attributed to the friction force on the tube wall due to the viscosity.

Remark that, in all computations, the parameters, $\rho, g$, and $\mu$, and the initial conditions, $P_{i}(0), Q_{i}(0)$, and $r_{i}(0)$, are fixed as in Table 1 . Other parameters and initial conditions of dependent variables such as $h_{i}(0), H_{L}(0)$, and $H_{R}(0)$ can be altered in various cases.

[Energy conserving property] The energy conserving is very important for stability of the model. In our system, several types of energy are involved, for example, the kinetic energies of the fluid in tube and tanks, the elastic potential energy of the tube, the external pumping energy, and the loss energy due to the viscosity. We show that these energies are balanced. In particular, the elastic energy turns out to be an essential part for the balancing.

The focus from now on is laid on the energy conservation property associated with the elastic energy of the vibrating tube. It is the main feature to understand how the elastic power generated by the expansion or contraction of the elastic tube transfers to the power acting on the fluid. In fact, during the derivation of the ECCM, the elastic energy is implicitly considered via the transformed fluid pressure. Since the mass of the elastic tube is assumed negligible in the model, the elastic energy consists of only a potential energy.

To explore how the elastic energy acts on the fluid pressure, we consider the kinetic energy of the fluid inside the tube, the potential energy of the elastic 
tube, the sum of the kinetic and potential energies of the fluid in both tanks, which are denoted by $e^{\text {fluid }}(t), e^{\text {elastic }}(t), e_{L}^{\operatorname{tank}}(t)$, and $e_{R}^{\operatorname{tank}}(t)$, respectively, at time $t$. Then, these energies are expressed as follows:

$$
\begin{aligned}
e^{f l u i d}(t) & =\frac{1}{2} \rho Q_{0}^{2}(t) \bar{\Delta}_{0}+\sum_{i=1}^{n-1} \frac{1}{2} \rho Q_{i}^{2}(t) \bar{\Delta}_{i}(t)+\frac{1}{2} \rho Q_{n}^{2}(t) \bar{\Delta}_{n}, \\
e^{\text {elastic }}(t) & =\sum_{i=0}^{n-1} \int_{0}^{t} P_{i}^{w}(\tau)\left(2 \pi r_{i}(\tau) l_{i}\right) \dot{r}(\tau) d \tau, \\
e_{L}^{\text {tank }}(t) & =\frac{1}{2} \rho Q_{0}^{2}(t) \Delta_{L}+\frac{1}{2} \rho g A_{L} H_{L}^{2}(t), \\
e_{R}^{\text {tank }}(t) & =\frac{1}{2} \rho Q_{n}^{2}(t) \Delta_{R}+\frac{1}{2} \rho g A_{R} H_{R}^{2}(t),
\end{aligned}
$$

where we define symbols

$$
\bar{\Delta}_{0} \equiv \frac{1}{2} \frac{V_{0}}{A_{0}^{2}}, \bar{\Delta}_{n} \equiv \frac{1}{2} \frac{V_{n-1}}{A_{n-1}^{2}} .
$$

It should be noted that the elastic power induced from the energy in (56) can be calculated as

$$
\begin{aligned}
\frac{d}{d t} e^{e l a s t i c} & =\sum_{i=0}^{n-1} 2 \pi r_{i} \dot{r}_{i} l_{i} P_{i}^{w}(t)=\sum_{i=0}^{n-1} P_{i}^{w}(t) \frac{d}{d t}\left(\pi l_{i} r_{i}^{2}(t)\right) \\
& =\sum_{i=0}^{n-1}\left(Q_{i}-Q_{i+1}\right)\left(P_{i}(t)-P^{a t m}-P_{i}^{e x t}(t)\right) \\
& =-\left(Q_{0}-Q_{n}\right) P^{a t m}+\sum_{i=0}^{n-1}\left(Q_{i}-Q_{i+1}\right)\left(P_{i}-P_{i}^{e x t}\right)
\end{aligned}
$$

On the other hand, referring to the identities, (8), (23), and (24), the energy involving the entire fluid becomes

$$
\begin{aligned}
& \frac{d}{d t}\left(e^{\text {fluid }}+e_{L}^{\text {tank}}+e_{R}^{\text {tank}}\right) \\
= & \left(-P_{0}+P^{a t m}\right) Q_{0}+\sum_{i=1}^{n-1}\left(P_{i-1}-P_{i}\right) Q_{i}+\left(P_{n-1}-P^{a t m}\right) Q_{n} \\
& -\left[8 \pi \mu Q_{0}^{2}(t) \frac{\bar{\Delta}_{0}}{A_{0}}+\sum_{i=1}^{n-1} 8 \pi \mu Q_{i}^{2}(t) \frac{\bar{\Delta}_{i}}{\bar{A}_{i}}+8 \pi \mu Q_{n}^{2}(t) \frac{\bar{\Delta}_{n}}{A_{n-1}}\right] \\
= & \left(Q_{0}-Q_{n}\right) P^{a t m}-\sum_{i=0}^{n-1}\left(Q_{i}-Q_{i+1}\right) P_{i}-\sum_{i=0}^{n} 8 \pi \mu Q_{i}^{2}(t) \frac{\bar{\Delta}_{i}}{\bar{A}_{i}},
\end{aligned}
$$


provided we identify $\bar{A}_{0}=A_{0}$ and $\bar{A}_{n}=A_{n-1}$. Therefore, if $e^{\text {total }}$ is assumed to be the total energy of this system including elastic energy, i.e.,

$$
e^{\text {total }} \equiv e^{\text {fluid }}+e^{\text {elastic }}+e_{L}^{\text {tank }}+e_{R}^{\text {tank }},
$$

then it satisfies the following energy balance at time $t$

$$
\dot{e}^{\text {total }}(t)=\underbrace{-\sum_{i=0}^{n-1}\left(Q_{i}-Q_{i+1}\right) P_{i}^{e x t}(t)}_{\text {power done by pumping }} \underbrace{-\sum_{i=0}^{n} 8 \pi \mu Q_{i}^{2}(t) \frac{\bar{\Delta}_{i}}{\bar{A}_{i}}}_{\text {power done by friction }} .
$$

Practically, the external pressure $P_{i}^{e x t}(t)$ is applied on a combination of compartments, for example, $C^{\text {pump }}=\bigcup_{k \in \Lambda^{e x t}} C_{k}$ for some compartment index set $\Lambda^{\text {ext }} \subset\{0,1, \ldots, n-1\}$, so that we have in this case

$$
\dot{e}^{t o t a l}(t)=-\sum_{k \in \Lambda^{e x t}}\left(Q_{k}-Q_{k+1}\right) P_{k}^{e x t}(t)-\sum_{i=0}^{n} 8 \pi \mu Q_{i}^{2}(t) \frac{\bar{\Delta}_{i}}{\bar{A}_{i}} .
$$

In the energy balance equation (63), the external pumping pressure $P_{i}^{\text {ext }}(t)$ on $C_{i}$ adds the power to the fluid inside $C_{i}$ in the manner of $\left(Q_{i}-Q_{i+1}\right) P_{i}^{\text {ext }}(t)$. Therefore, the total energy added to the system by the external pumping can be calculated by using the equation

$$
\dot{e}^{\text {pump }}=\sum_{i=0}^{n-1}\left(Q_{i}-Q_{i+1}\right) P_{i}^{e x t}(t) .
$$

Contrary to the pumping power, there exists the friction power appearing at the last term in (63), which is always negative to cause the energy loss. By the energy conserving in the ECCM, we mean that the entire energy of the system including the energy loss by the friction power is conserved in the sense that, if we define the friction energy

$$
\dot{e}^{\text {fric }}=\sum_{i=0}^{n} 8 \pi \mu Q_{i}^{2}(t) \frac{\bar{\Delta}_{i}}{\bar{A}_{i}}
$$

then we obtain the energy conservation

$$
\frac{d}{d t}\left(e^{\text {fluid }}+e^{\text {elastic }}+e_{L}^{\text {tank }}+e_{R}^{\text {tank }}+e^{\text {pump }}+e^{\text {fric }}\right)=0 .
$$

\section{Numerical results on valveless pumping effects}

To make the valveless pumping power available and controllable to mechanical devices, what we have to do first is to understand why a preferential direction of the net power occurs at a specific frequency of the periodic external pumping. Assume that the external pumping $P_{k}^{e x t}$ is applied on the compartments $C_{k}$ 's for $k \in \Lambda^{e x t}$ for a pumping index set $\Lambda^{e x t} \subset\{0,1, \ldots, n-1\}$ which indicates the pumping region. If the pumping region index $\Lambda^{\text {ext }}$ is given, then, as an external pumping on $\Lambda^{\text {ext }}$, the following type of time dependent periodic non-negative function with frequency $\omega$ is employed: 
TABLE 2. Parameters and initial conditions for computing $H_{R}-H_{L}$ as the frequency $\omega$ ranges from $0[\mathrm{~Hz}]$ to $60[\mathrm{~Hz}]$.

\begin{tabular}{c|l} 
parameter & value [CGS unit] \\
\hline$n$ & 20 Compartments \\
$L$ & 40 \\
$E$ & $1.0 \times 10^{7}$ \\
$d_{L}=d_{R}$ & 5 \\
$H_{L}(0)=H_{R}(0)$ & 20 \\
$r_{i}(0)$ & 0.5 \\
$h_{i}(0)$ & 0.1 \\
$P^{\text {max }}$ & $1.01325 \times 10^{5}\left(=\frac{P^{\text {atm }}}{10}\right)$ \\
\hline
\end{tabular}

$$
P_{k}^{e x t}(t) \equiv \begin{cases}\frac{P_{\max }}{2}(1-\cos (2 \pi \omega t)), & \text { for all } k \in \Lambda^{\text {pump }}, \\ 0, & \text { otherwise. }\end{cases}
$$

If a function $f(t)$ fluctuate at sufficiently large time, we define its asymptotic average as

$$
\overline{f(t)}=\lim _{t \rightarrow \infty} \frac{1}{T} \int_{t}^{t+T} f(\tau) d \tau,
$$

where $T=1 / \omega$ is the period of time. Assume $H_{L}$ and $H_{R}$ are the asymptotic averages of $H_{L}(t)$ and $H_{R}(t)$, respectively. When using the external pumping function given in (68) and parameters in Table 2, the influences on $H_{R}-H_{L}$ by the pumping location and the width of pumping region are shown in Fig. 6 . The external pumping is located at some compartments on the left half of the elastic tube. The behavior of $H_{R}-H_{L}$ has no consistency for pumping locations (see Fig. 6(a)) while there appears a similar pattern of the difference $H_{R}-H_{L}$ for the variation of the width of the pumping region (see Fig. 6(b)). Therefore, we can anticipate that the pumping location is more important factor for valveless pumping than the width of pumping region. Among these cases, we choose one case where $C^{\text {pump }}=C_{2} \cup C_{3}$ to study in detail the features of producing a net power in two open tanks connected by the elastic tube. Fig. 7 shows the graph of $H_{R}-H_{L}$ in the chosen case on which the peaks are numbered from (1) to (10).

It is well observed that either direction for energy storing can take place in this figure. Of more interest is the graph in Fig. 8 which illustrates the stored energy in asymptotic average, $\frac{1}{\alpha}\left(\overline{e_{R}^{\operatorname{tank}}(t)}-\overline{e_{L}^{\operatorname{tank}}(t)}\right)$ for some scaling constant $\alpha$. The scaling factor $\alpha$ is chosen inspired by the average potential energy difference of two tanks

$$
\alpha=\frac{1}{2} g A_{L}\left(H_{L}+H_{R}\right) .
$$


Then, as seen in Fig. 8, the stored potential energy is proportional to $H_{R}-$ $H_{L}$. It can be a salient feature that $H_{R}-H_{L}$ and the stored energy is perfectly correlated. However, it is not obvious since the difference of average energy in two tanks are induced from the energy equations, (57) and (58). According to the computational results, it is obvious that

$$
H_{R}-H_{L}=\frac{1}{\alpha}\left(e_{R}^{\operatorname{tank}}-e_{L}^{\operatorname{tank}}\right)
$$

where $e_{L}^{\text {tank }}$ and $e_{R}^{\text {tank }}$ mean the asymptotic average of $e_{L}^{\operatorname{tank}}(t)$ and $e_{R}^{\operatorname{tank}}(t)$, respectively.

While inspecting the factor related to the direction of net power using numerical experiments, we find out that the fluid pressure difference, particularly over the pumping region, plays an important role in determining the direction of net power to store the energy in the direction of a preferential tank. For the purpose of proposing a quantitative index to judge the net power direction including its magnitude, we define the pressure difference of fluids in $C_{k}$ induced from the external pumping $P_{k}^{e x t}$ as shown in Fig. 9:

$$
(\Delta P)_{k}(t) \equiv \int_{C_{k}} P_{x} d x=\bar{P}_{k+1}(t)-\bar{P}_{k}(t),
$$

where $\bar{P}_{k}(t)$ means the fluid pressure interpolation at the site where $Q_{k}$ is defined and it should be defined with energy conserving on the control volume $\bar{V}_{k}$ at the position of $Q_{k}$ such that

$$
\bar{P}_{k}(t) \bar{V}_{k}(t)=P_{k-1}(t)\left(\frac{1}{2} V_{k-1}(t)\right)+P_{k}(t)\left(\frac{1}{2} V_{k}(t)\right) .
$$

An index $D^{\text {power }}$ correlated to the net pumping power is defined as the asymptotic average of the sum of $(\Delta P)_{k}(t) \times P_{k}^{e x t}(t)$ over the pumping compartments:

$$
D^{\text {power }} \equiv \overline{\sum_{k \in \Lambda^{e x t}}(\Delta P)_{k}(t) P_{k}^{e x t}(t)}
$$

In our case, since $P_{k}^{e x t}(t)$ for any pumping index $k$ is the same function of $t, P^{e x t}(t)$, as shown in (68), we have

$$
D^{\text {power }}=\overline{\Delta P(t) P^{e x t}(t)}
$$

where $(\Delta P)(t)=\sum_{k \in \Lambda^{e x t}}(\Delta P)_{k}(t)$.

The meaning of the index $D^{\text {power }}$ can be viewed as the phase synchronization between $\Delta P$ and $P^{e x t}$. In other words, if the excitation timing on the pumping region synchronizes with the pressure difference on it, then the net power becomes positive. On the contrary, if the skew-synchronization occurs, the negative power takes place. Numerical computations shows well these features as illustrated in Fig. 10. A series of graphs at 10 peaks are plotted consecutively. The odd columns represent graphs of the phase synchronization between $(\Delta P)(t)$ and $P^{e x t}(t)$ at every peak numbered with (1) through (10) and the graphs in even columns show the multiplication of corresponding two factors, 

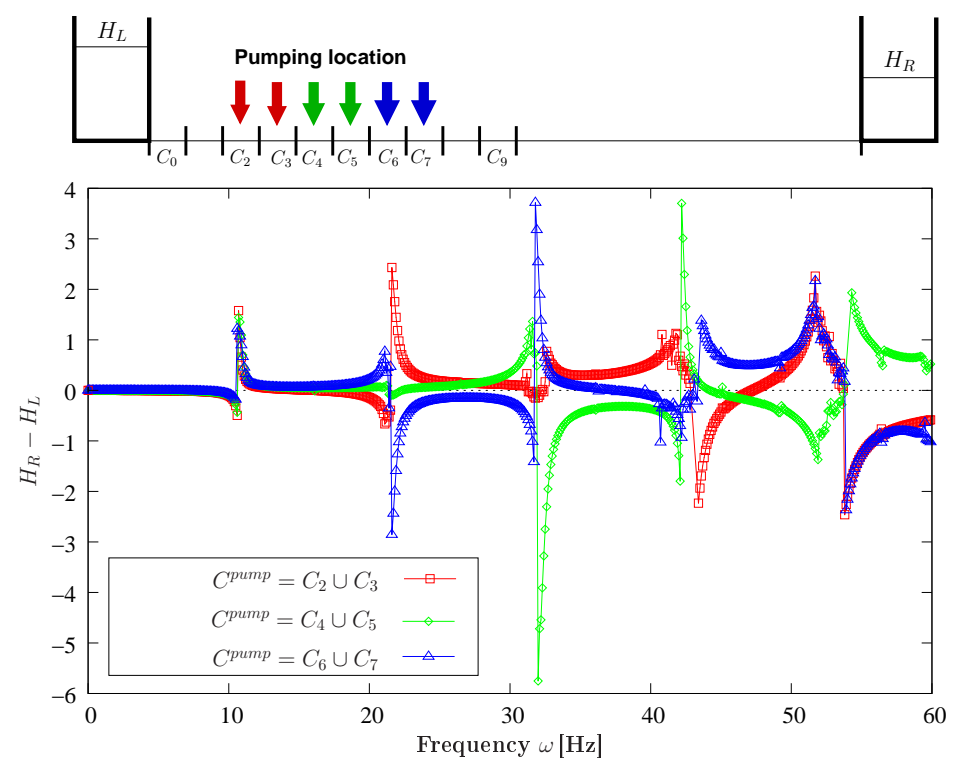

(a) The effect by the pumping location

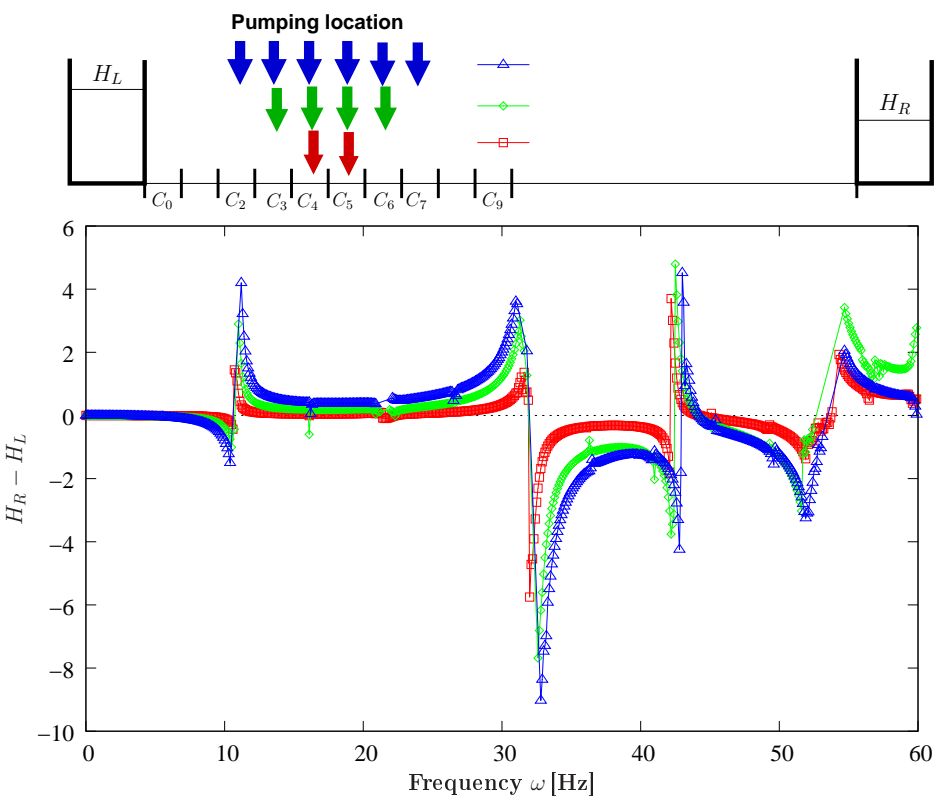

(b) The effect by the width of pumping region

FiguRE 6 . Influences on $H_{R}-H_{L}$ by the pumping locations and the width of pumping region. 


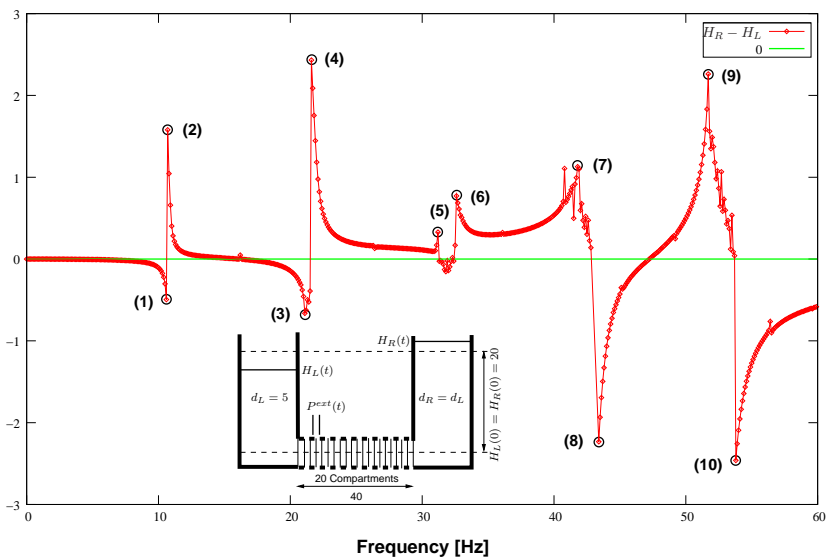

FiguRE 7. $H_{R}-H_{L}$ as the pumping frequency $\omega$ varies when pumping region is the compartments $C^{\text {pump }}=C_{2} \cup C_{3}$.

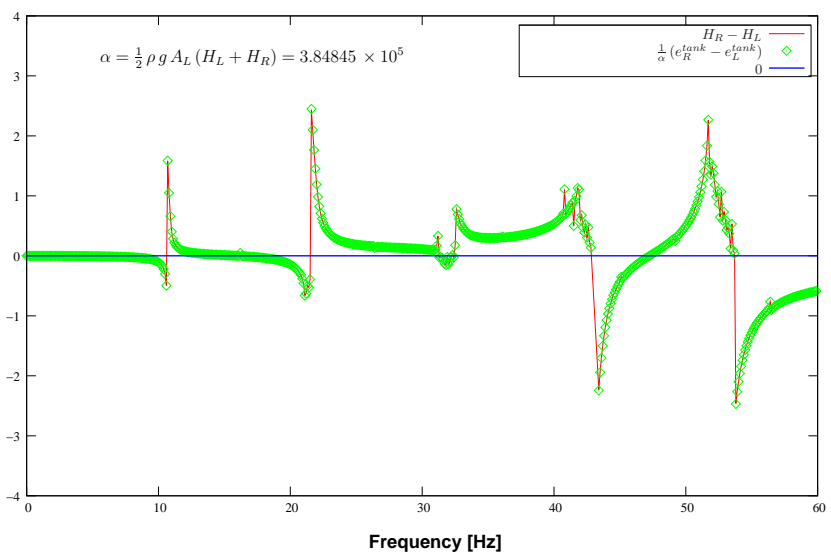

FiguRE 8. The relationship between $\frac{1}{\alpha}\left(e_{R}^{\text {tank }}-e_{L}^{\text {tank }}\right)$ and $H_{R}-H_{L}$ when pumping region is the compartments $C^{\text {pump }}=$ $C_{2} \cup C_{3}$.

$(\Delta P)(t)$ and $P^{e x t}(t)$, to view the size of signed area above and below zero. In Table 3 , the sign and magnitude of $D^{\text {power }}$ indicate the direction and strength of the net power, respectively, at the tagged peaks except the transient states (5) and (7) in Fig. 7. The index $D^{\text {power }}$ can be also interpreted as a kind of covariance in time between $\Delta P(t)$ and the external pumping $P^{e x t}(t)$ on the pumping compartments. Consequently, the phase synchronization of $(\Delta P)(t)$ with the external pumping is an important factor to produce and maintain the directional net power. 


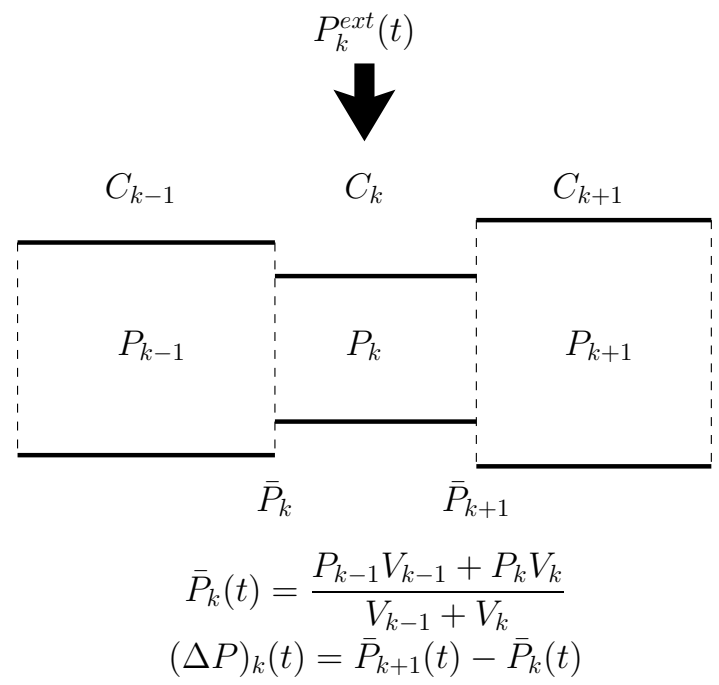

FiguRE 9. The definition of pressure difference $(\Delta P)_{k}$ is illustrated on the $k$-th compartment $C_{k}$ to which the external pumping $P_{k}^{e x t}(t)$ is applied. The average of the product $(\Delta P)_{k}(t) P_{k}^{e x t}(t)$ constitutes the index $D^{\text {power }}$.

TABle 3. Comparison of the index $D^{\text {power }}$ and $H_{R}-H_{L}$ at each peak.

\begin{tabular}{c|c|l|l} 
peak number & external pumping frequency $(\omega)$ & index $D^{\text {power }}\left[\times 10^{9}\right]$ & $H_{R}-H_{L}$ \\
\hline $\mathbf{( 1 )}$ & 10.6 & -1.0143 & -0.4947 \\
$\mathbf{( 2 )}$ & 10.7 & +2.1062 & +1.5794 \\
$\mathbf{( 3 )}$ & 21.1 & -1.4345 & -0.6632 \\
$\mathbf{( 4 )}$ & 21.6 & +2.7677 & +2.4324 \\
$\mathbf{( 5 )}$ & 31.2 & -0.0579 (transient) & +0.2842 \\
$\mathbf{( 6 )}$ & 32.6 & +0.7643 & +0.7718 \\
$\mathbf{( 7 )}$ & 41.8 & +2.7812 (transient) & +1.0849 \\
$\mathbf{( 8 )}$ & 43.4 & -2.7266 & -2.2331 \\
$\mathbf{( 9 )}$ & 51.7 & +4.0763 & +2.2615 \\
$\mathbf{( 1 0 )}$ & 53.8 & -3.6763 & -2.4610 \\
\hline
\end{tabular}

\section{Conclusion}

To understand the valveless pumping phenomena, we have developed the energy conserving compartment model (ECCM) based on the energy principle. Computations including reliability tests for the ECCM are performed in various cases. It is our numerical conclusion for the valveless pumping effect that the energy stored in the valveless pumping system under gravity is attributed to 

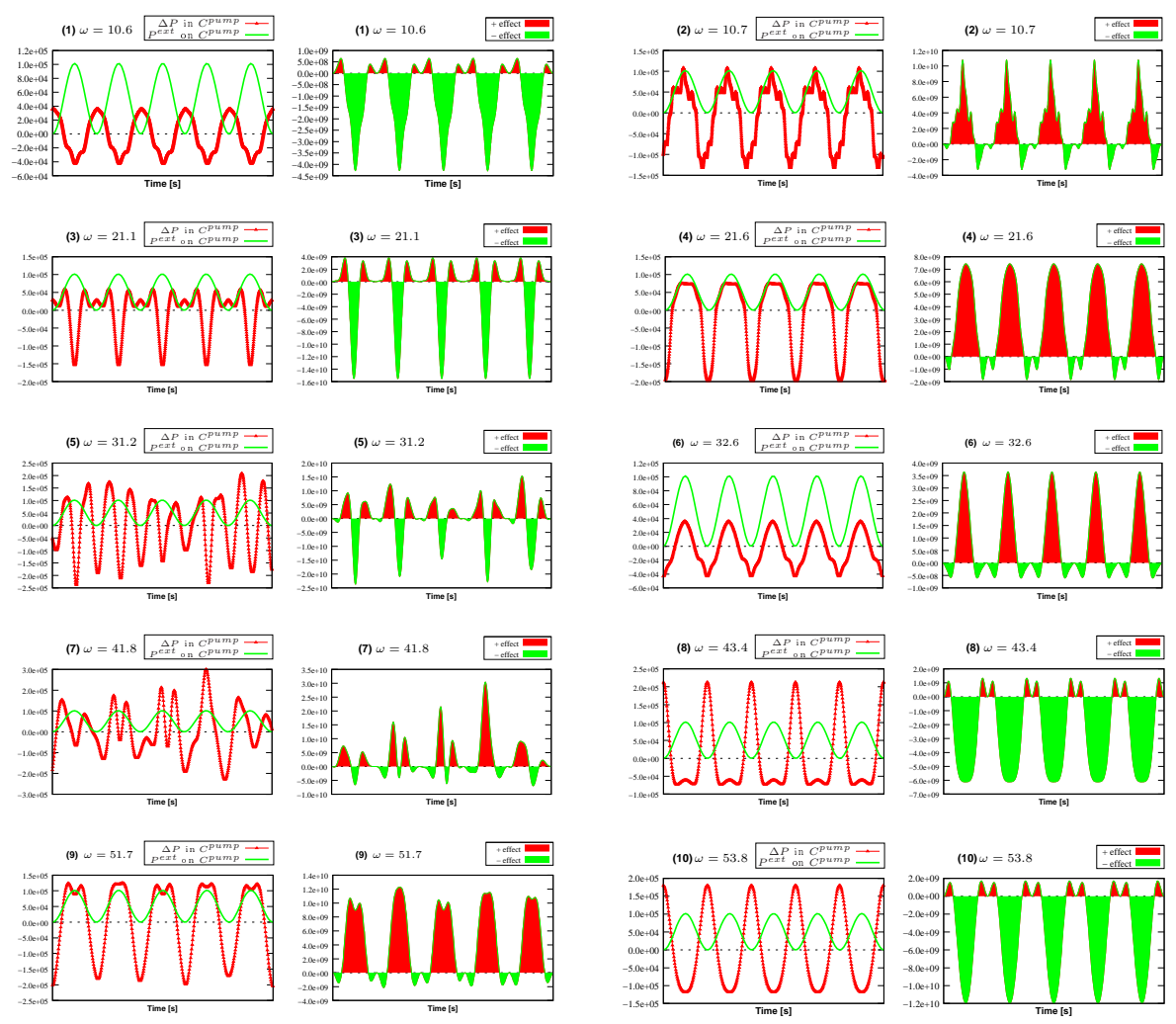

Figure 10. Phase synchronization between the pressure difference $(\Delta P)(t)$ and external pumping $P^{e x t}(t)$ on the pumping location $C^{\text {pump }}$ in cases tagged with (1) to (10) in Fig. 7.

the directional net power caused by the simple external pumping on the elastic tube. Moreover, it is also revealed that the stored energy is proportional to the difference of asymptotic average levels of fluid in two tanks. Finally, an indicator for anticipating the direction of net power generated by the external pumping is discovered. It is closely related to the direction of the net power as well as its magnitude. In fact, the indicator proposed can be regarded as a kind of phase synchronization or covariance between the pressure difference in time and the external pumping wave over the pumping region. Synchronized phases trigger the positive net power (from the left tank to the right) while anti-synchronized phases generate the negative net power (from the right tank to the left). With the computationally discovered facts, we want to control the power of the valveless pumping. Perfectly controlling will enable us to develop the micro-machine. 


\section{References}

[1] D. Auerbach, W. Moehring, and M. Moser, An analytic approach to the Liebau problem of valveless pumping, Cardiovasc. Eng. 4 (2004), 201-207.

[2] I. Avrahami and M. Gharib, Computational studies of resonance wave pumping in compliant tube, J. Fluid Mech. 608 (2008), 139-160.

[3] A. Borzi and G. Propst, Numerical investigation of the Liebau phenomenon, Z. Angew. Math. Phys. 54 (2003), no. 6, 1050-1072.

[4] T. T. Bringley, S. Childress, N. Vandenberghe, and J. Zhang, An experimental investigation and a simple model of a valveless pump, Phys. Fluids 20 (2008), 033602.

[5] H.-T. Chang, C.-Y. Lee, and C.-Y. Wen, Design and modeling of a MEMS-based valveless pump driven by an electromagnetic force, DTIP of MEMS \& MOEMS, Stresa, Italy, 26-28 April 2006.

[6] H.-T. Chang, C.-Y. Lee, C.-Y. Wen, and B.-S. Hong, Theoretical analysis and optimization of electromagnetic actuation in a valveless microimpedance pump, Microelectron. J. 38 (2007), 791-799.

[7] A. I. Hickerson, An Experimental Analysis of the Characteristic Behaviors of an Impedance Pump, Thesis, California Institute of Technology Pasadena, California, 2005.

[8] A. I. Hickerson, D. Rinderknecht, and M. Gharib, Experimental study of the behavior of a valveless impedance pump, Exps. Fluids 38 (2005), 534-540.

[9] E. Jung, A mathematical model of valveless pumping: A lumped model with timedependent compliance, resistance, and inertia, Bull. Math. Biol. 69 (2007), no. 7, 21812198.

[10] T. Kenner, Biological asymmetry and cardiovascular blood transport, Cardiovasc. Eng. 4 (2004), 209-218.

[11] J. Koo and C. Kleinstreuer, Viscous dissipation effects in microtubes and microchannels, Int. J. Heat Mass Tran. 47 (2004), 3159-3169.

[12] S. Lee and E. Jung, A two-chamber model of valveless pumping using the immersed boundary method, Appl. Math. Comput. 206 (2008), no. 2, 876-884.

[13] W. Lee, E. Jung, and S. Lee, Simulations of valveless pumping in an open elastic tube, SIAM J. Sci. Comput. 31 (2009), no. 3, 1901-1925.

[14] G. Liebau, Prinzipien Kombinierter Ventilloser Pumpen, Abgeleitet Vom Menschlichen Blutkreislauf, Naturwissenschaften 42 (2008), 339.

[15] Y. Y. Lin Wang, W.-B. Chiu, M.-Y. Jan, J.-G. Bau, S.-P. Li, and W.-K. Wang, Analysis of transverse wave as a propagation mode for the pressure pulse in large arteries, J. Appl. Phys. 102 (2007), 064702.

[16] L. Loumes, I. Avrahami, and M. Gharib, Resonant pumping in a multilayer impedance pump, Phys. Fluids 20 (2008), 023103

[17] C. G. Manopoulos, D. S. Mathioulakis, and S. G. Tsangaris, One-dimensional model of valveless pumping in a closed loop and a numerical solution, Phys. Fluids 18 (2006), 017106.

[18] C. G. Manopoulos and S. Tsangaris, Modelling of the blood flow circulation in the human foetus by the end of the third week of gestation, Cardiovasc. Eng. 5 (2005), 29-35.

[19] A. Olsson, G. Stemme, and E. Stemme, A numerical design study of the valveless diffuser pump using a lumped-mass model, J. Micromech. Microeng. 9 (1999), 34-44.

[20] J. T. Ottesen, Valveless pumping in a fluid-filled closed elastic tube-system: onedimensional theory with experimental validation, J. Math. Biol. 46 (2003), no. 4, 309332.

[21] G. Propst, Pumping effects in models of periodically forced flow configurations, Phys. D 217 (2006), no. 2, 193-201.

[22] D. Rinderknecht, A. I. Hickerson, and M. Gharib, A valveless micro impedance pump driven by electromagnetic actuation, J. Micromech. Microeng. 15 (2005), 861-866. 
[23] S. Takagi and K. Takahashi, Study of a piston pump without valves, Bull. JSME 28 (1985), 831-836.

[24] S. Timmermann and J. T. Ottesen, Novel characteristics of valveless pumping, Phys. Fluids 21 (2009), 053601.

[25] A. Ullmann and I. Fono, The Piezoelectric Valve-Less Pump-Improved Dynamic Model, J. Microelectromech. Syst. 11 (2002), 655-664.

EUNOK Jung

Department of Mathematics

KONKUK UNIVERSITY

SeOul 143-701, Korea

E-mail address: junge@konkuk.ac.kr

Do Wan KIM

Department of Mathematics

INHA UNIVERSITY

INCHEON 402-751, KOREA

E-mail address: dokim@inha.ac.kr 